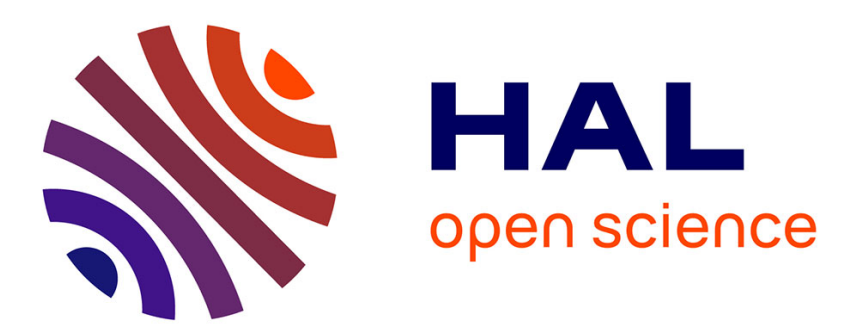

\title{
Frequency-dependent dielectric permittivity of salt-free charged lamellar systems
}

Benjamin Rotenberg, Jean-François Dufrêche, Pierre Turq

\section{To cite this version:}

Benjamin Rotenberg, Jean-François Dufrêche, Pierre Turq. Frequency-dependent dielectric permittivity of salt-free charged lamellar systems. Journal of Chemical Physics, 2005, 123, pp.154902. hal00016443

\section{HAL Id: hal-00016443 \\ https://hal.science/hal-00016443}

Submitted on 9 Nov 2018

HAL is a multi-disciplinary open access archive for the deposit and dissemination of scientific research documents, whether they are published or not. The documents may come from teaching and research institutions in France or abroad, or from public or private research centers.
L'archive ouverte pluridisciplinaire HAL, est destinée au dépôt et à la diffusion de documents scientifiques de niveau recherche, publiés ou non, émanant des établissements d'enseignement et de recherche français ou étrangers, des laboratoires publics ou privés. 


\title{
Frequency-dependent dielectric permittivity of salt-free charged lamellar systems
}

\author{
B. Rotenberg*, J.-F. Dufrêche and P. Turq
}

June 10, 2005

Laboratoire Liquides Ioniques et Interfaces Chargées (UMR CNRS 7612), Université P. et M. Curie, 4 place Jussieu, 75252 Paris Cedex 05, France

\begin{abstract}
We present a new model to analyze dielectric spectroscopy measurements on charged lamellar systems, with the following improvements with respect to the hitherto available models: (i) it does not rely on the hypothesis of local electroneutrality, and allows to treat the salt-free case; (ii) the chemical exchange governing the partition between free and bound ions is properly taken into account; (iii) a fully analytical solution is provided. The variation of the frequency-dependent dielectric permittivity with both thermodynamic and kinetic characteristics of the free-bound ion equilibrium is presented. In particular, the relative weights of both relaxation modes (exchange and transport), and their characteristic frequencies are discussed. This study opens the way to the analysis of systems for which the usual models are irrelevant, such as salt-free clay gels or membranes.
\end{abstract}




\section{Introduction}

The dielectric properties of heterogeneous materials consisting of inclusions of a conducting medium in an insulating matrix have attracted the attention of scientists for more than a century[1]. Early theories, known as Maxwell-Wagner[2] and later MaxwellWagner-Sillars[3] theories were based solely on macroscopic electrodynamics. Later on, considerable effort has been devoted to developing theoretical models based on a microscopic description of the charge carriers in the conducting inclusions, and the complex permittivity could be related with some success to the motion of charge carriers. In particular, its frequency dependence was derived from the dynamics of charge relaxation, either directly in the conducting medium, often an electrolyte consisting of anions and cations dispersed in a solvent $[4,5]$, or from the relaxation of charges accumulated at its boundaries $[6,7,8,9,10,11,12,13,14,15]$. The relaxation of the ionic cloud in an electrolyte was first described by Debye, and its peculiar frequency dependence is now known as the Debye-Falkenhagen effect[4]. More recently, our understanding of the frequency dependent conductivity of bulk electrolytes has been improved by the use of Mode Coupling Theory[5]. In solid ionic conductors too, the concepts of ionic atmosphere and of its relaxation seem to be appropriate to interpret the frequency-dependent conductivity[16]. Charge accumulation at boundaries in presence of an applied AC electric field was largely discussed in various contexts such as materials between (blocking or partially non-blocking) electrodes $[6,7,8]$, saline inclusions in rocks[9] or lamellar systems[10, 11, 12], particularly biological membranes.

Though often described in terms of equivalent electrical circuits, the frequency dependent conductivity of electrolytes, colloidal suspensions or emulsions, can be efficiently interpreted on a more physical basis at the Smoluchowski level by assuming that the transport of the charged species is governed by the Nernst-Planck equation[13, 14]. The pioneering work of Trukhan[15] in the context of conducting inclusions in an insulating material, both in planar and spherical geometries, opened the way to the study of lamellar systems $[10,11,12]$. The resolution of the problem is in the previous cases greatly 
simplified by assuming that electro-neutrality is locally satisfied, in the absence of the applied field. Space charges then arise only near the electrodes, under the influence of the field. This picture is only justified when the Debye screening length, characterizing the range of electrical interactions, is much smaller than the system size. In the electrochemical context, this is achieved by the presence of a "supporting electrolyte". Even in presence of an electrolyte, recent studies have shown that under an alternating field, electro-neutrality could be perturbed over distances much larger than the Debye length if the diffusion coefficients of anions and cations are different, in dramatic contrast with the results for a DC field $[17,18]$. The authors were able to provide a fully analytical solution for insulating spheres suspended in an electrolyte[17], and numerical results for charged particles[18]. Two important conclusions are: (i) AC fields can induce violations of electro-neutrality over large distances, resulting in substantial contributions to the dielectric behavior of the suspension, even for systems that are neutral at equilibrium; (ii) the effect can be up to five orders of magnitude larger for charged particles, i.e. when a space charge is present at equilibrium.

From the theoretical point of view, all investigations of the polarization (either at electrodes or of lamellar systems) have assumed so far that the system under consideration was locally electro-neutral. In many systems however, when no "supporting electrolyte" is added, charges arising from the dissociation of surface sites, either acidic or cation compensated sites (such as oxygen atoms of mica or clay sheets, or the hydrophilic head of an ionic surfactant) can be found in the vicinity of the charged surfaces, mobile in the solvent which thereby becomes a region of space charge, and an internal electric field arises. Electro-neutrality is in that case only guaranteed by the presence of the (oppositely) charged surfaces. The equilibrium profile of the counter-ion distribution depends on the charge density of the surface, and is determined by the compensation of diffusion (due to concentration gradients) and migration (due to the internal electric field): it leads to the well-known Poisson-Boltzmann solution[19, 20]. The dynamical response to an applied field of this charge distribution was apparently never studied, though it 
is necessary if one wants to investigate the permittivity of such systems, which contain no added salt. A possible reason for this absence of consideration might be found in the difficulty of the task. Indeed, the coupling of this distribution with an electric field does include a contribution which is absent for locally neutral system, namely that of the internal electric field "at rest".

On the experimental side, many systems belonging to this category (no added salt) have received over the last decades a growing interest. In particular, the dynamics of cations in clays was recognized as a crucial point because these materials are considered as possible (engineered and geological) barriers for the long term storage of nuclear wastes disposal[21]. Among other studies, dielectric spectroscopy has proved very promising. However, there is a need for a theoretical background to interpret the experimental data and extract microscopic information from these macroscopic measurements. The hitherto available models are restricted to the case of added salt and are therefore not relevant for this purpose.

Another important feature of the ionic dynamics, which concerns all the above-mentioned cases, is the possibility of adsorption of the ions at the surfaces. This adsorption is generally ion-specific (not only charge-dependent), and both the chemical equilibrium at the surfaces can influence the dynamics of the charge relaxation in the liquid phase. Indeed, in bulk electrolytes, it has been shown that chemical association between ions could influence the charge dynamics[22], while relaxation of the ionic cloud do influence diffusion limited reactions[23]. Similarly bound (to a surface) and free ions don't have the same mobility (the latter being zero for bound ions), so that the exchange between both forms affects the overall dynamics of the mobile ions. The influence of such an exchange on dynamics has been reported in the case of water in clays[24], and especially on dielectric properties for exchangeable polar molecules in general[25] and for water bound to biological molecules[26] in particular. The exchange of cations is something different, but a parallel can be drawn with these studies, since it also affects the dynamics, and hence the dielectric properties. In micellar solutions, the exchange between cations 
bound to the surface (immobile) with others diffusing around the micelles was shown to lead to a complex dielectric response[27, 28]. In turn, one can ask if it is possible to extract the thermodynamic (distribution constant between bound and free ions) and dynamic (kinetic constants of the adsorption and desorption reaction) characteristics of the exchange reaction from the dielectric permittivity of the whole system, which is a probe of the global charge dynamics.

The scope of the present paper is to show that despite the complexity of the problem, a (relatively) simple analytical solution can be found in the case where charged species in the liquid phase stem only from dissociation of the surfaces, and when a chemical equilibrium dictates the partition between free and bound ions. The solution is presented and the influence of structural and kinetic parameters on the complex permittivity is discussed. The possibility of extracting microscopic information from its experimental determination is also considered. The paper is organized as follows. In section 1, the microscopic model is presented. In section 2, the equilibrium concentration profile and internal electric field are recalled. Section 3 presents the fully analytical solution for the complex permittivity of the system; the resulting expression, particularly the influence of the various parameters, is discussed in section 4. Finally, section 5 summarizes the major findings of the present work.

\section{Microscopic model}

We only consider the ionic motion normal to the charged surfaces. Within the meanfield treatment adopted here, there is no concentration gradient parallel to the surfaces, and therefore no diffusive flux (nor internal electric field) along the planes $\left(E_{\|}=0\right)$. We restrict ourselves to the homo-ionic case (i.e. only one type of counter-ions), with counterions characterized by their charge $z$ and their diffusion coefficient $D$. We also restrict ourselves to the case of no added salt: the only ions between the charged surfaces come from the dissociation of surface sites. Electro-neutrality is guaranteed by the presence of 
the charged surfaces.

The ionic distribution is described in terms of a two-state model: ions can be either adsorbed on the surfaces (Stern layer), or mobile in the interlayer space. Such a distribution is depicted in figure 1. This model accounts for the possible formation of inner-sphere complexes at the surface, where some solvent (water) molecules of the ion's coordination shell are replaced by atoms of the charged surface. Such a distribution is characterized by the concentration profile $c(x, t)$ (in $m^{-3}$ ), and the adsorption on both surfaces $\Gamma_{ \pm}(t)$ (in $\left.m^{-2}\right) . \Gamma_{+}\left(\operatorname{resp} . \Gamma_{-}\right)$refers to the surface at $x=+L / 2($ resp. $x=-L / 2)$, where the origin of the $x$ axis normal to the surfaces has been taken in the middle of the interlayer space ( $L$ is the interlayer distance).

A particular ion located near a charged surface (at $x= \pm L / 2$ ), can switch between the two states following first order kinetics, i.e. the number of mobile ions adsorbing per unit surface and per unit time is $k_{1}^{\prime} c( \pm L / 2, t)\left(k_{1}^{\prime}\right.$ in $\left.m . s^{-1}\right)$, whereas the number of adsorbed ions desorbing per unit surface and per unit time is $k_{2} \Gamma_{ \pm}(t)\left(k_{2}\right.$ in $\left.s^{-1}\right)$. The prime is here to underline that $k_{1}^{\prime}$ and $k_{2}$ don't have the same dimensionality. At equilibrium, we simply have $k_{1}^{\prime} c_{0}( \pm L / 2)=k_{2} \Gamma_{0}$. The relaxation towards chemical equilibrium has a time constant:

$$
\tau_{\chi}=\frac{1}{k_{1}^{\prime} / L+k_{2}}
$$

One can define the distribution constant $K_{d}$ as the ratio between the number of bound ions per unit volume vs. mobile ions per unit volume.

$$
K_{d}=\frac{\text { bound }}{\text { mobile }}
$$

With this definition, $K_{d}$ is not the equilibrium constant of the exchange reaction. We will discuss later the relation between $K_{d}$ and the kinetic constants. The bare charge of the surface is $-\sigma_{0}$ (without loss of generality we consider that the counter-ions are cations, and thus $\left.\sigma_{0}>0\right)$, and their effective charge $-\sigma=-\sigma_{0}+z \Gamma_{0}=-\sigma_{0} /\left(1+K_{d}\right) . K_{d}$ will be shown to have a large influence on the dynamics of the charge distribution in the presence of an applied electric field, and hence on the polarization of the interlayer. 
The solvent is treated as a continuum of dielectric constant $\epsilon_{S}$, and all dielectric relaxation mechanisms associated with water, such as dipolar rotation of water molecules are therefore outside the scope of the present model. Their contribution to the dielectric spectrum should be added to the one that we are calculating here, and the dielectric constant of the whole interlayer is hence larger than that of the pure solvent (see section 4). This assumption makes sense if the relaxation time of the solvent (typical time for a solvent molecule reorientation) is much shorter than the relaxation time(s) of the ions. Indeed, in that case the two processes correspond to different time scales and can be considered as decoupled. Such an assumption should be verified a posteriori, from the experimental measurements. The value of $\epsilon_{S}$ is debatable, since for confined water (small $L)$, there is no clear reason for it to be equal to its bulk value. Nevertheless, there has been some evidence that a mesoscopic model such as Poisson-Boltzmann involving the bulk value of $\epsilon_{S}$ can provide reasonable results, at least as far as the equilibrium profile is concerned, for distances as short as $15 \AA[19]$.

\section{Equilibrium concentration profile}

The charge of the surfaces attracts the counter-ions, thereby enhancing their local concentration. This density inhomogenity gives rise to an internal electric field and a diffusive flux. At equilibrium, diffusive and migration (electric) fluxes cancel out. The total flux $j(x)$ is then identically zero:

$$
j(x)=-D \frac{\mathrm{d} c}{\mathrm{~d} x}+\frac{z D}{k_{B} T} c(x) E(x)=0
$$

where $E$ is the electrical field, deriving from the electrical potential $V(E=-\mathrm{d} V / \mathrm{d} x)$. $k_{B}$ is the Boltzmann constant, and $T$ the temperature. Furthermore, the local Gauss theorem reads:

$$
\frac{\mathrm{d} E}{\mathrm{~d} x}=-\frac{\mathrm{d}^{2} V}{\mathrm{~d} x^{2}}=\frac{z c(x)}{\epsilon_{0} \epsilon_{s}}
$$

Inserting (4) in (3), and using the electro-neutrality condition (the total number of ions compensates the total charge of the surfaces), one finds for the concentration profile the 
Poisson-Boltzmann solution[19, 20]:

$$
c_{0}(x)=\frac{1}{2 \pi L_{B}} \frac{\alpha^{2}}{\cos ^{2}(\alpha x)}
$$

with

$$
2 \pi L_{B} \frac{\sigma}{z}=\alpha \tan \frac{\alpha L}{2}
$$

where $L_{B}=z^{2} / 4 \pi \epsilon_{0} \epsilon_{s} k_{B} T$ is the Bjerrum length, and for the internal electric field:

$$
E_{0}(x)=\frac{z}{\epsilon_{0} \epsilon_{s}} \frac{\alpha}{2 \pi L_{B}} \tan (\alpha x)
$$

These quantities are both symmetric with respect to the $x=0$ plane, which means that $c_{0}(x)$ (scalar) is even while that $E_{0}(x)$ (component of a vector) is odd. The constant $\alpha$ can be interpreted as an inverse screening length, generalizing the Debye inverse screening length $\kappa$ to the case of a confined electrolyte. The dimensionless parameter $\alpha L$ hence determines the "softness" of the ionic profile (see equation (5)). Equation (6) implies that for a given bare charge density $\sigma_{0}, \alpha L$ implicitly depends on $K_{d}$ via the effective charge $\sigma$. Indeed, it can be rewritten as:

$$
\frac{\alpha L}{2} \tan \frac{\alpha L}{2}=\frac{L}{4 \lambda}=\frac{L}{4}\left(\frac{\sigma_{0} z}{\epsilon_{0} \epsilon_{S} k_{B} T} \times \frac{1}{1+K_{d}}\right)
$$

where one has introduced the $\left(K_{d}\right.$ dependent) Gouy length $\lambda$, defined as the distance from the charged plane at which the electrostatic energy of an ion is equal to the thermal energy (neglecting the influence of the other ions). The ratio $L / \lambda$ completely defines the parameter $\alpha L$, and hence the equilibrium profile. Figure 2a shows the evolution of $\alpha L$ with $L / \lambda$. For small charge densities $(L / \lambda \rightarrow 0)$ one has $\alpha L \sim(L / \lambda)^{1 / 2}$, while $\alpha L \rightarrow \pi$ for large values of $L / \lambda$. The concentration profiles corresponding to three values of the charge density have been reported in figure $2 \mathrm{~b}$. The values are chosen to reproduce typical charge density and interlayer spacing for a smectite clay at low water content $\left(\sigma_{0}=0.131 \mathrm{Cm}^{-2}\right.$, $\left.L=6 \AA, \epsilon_{S}=78\right)$, and three values of the distribution constant $K_{d}(0.1,1.0$ and 10$)$, at room temperature $\left(k_{B} T=4.10^{-21} \mathrm{~J}\right)$. The corresponding values of $L / \lambda$ and $\alpha L$ are summarized in table 1 . It is clear from figure $2 \mathrm{~b}$ that when $L / \lambda$ is small, the concentration 
profile is very flat, while for large values of $L / \lambda$ it is sharply increasing in the vicinity of the charged planes. Such different ionic distributions will respond very differently to an applied electric field, as will be shown in section 4 .

For the same lamellar system (same bare charge $\sigma_{0}$, same interlayer spacing $L$, same counter-ion charge $z$ ), the specific interactions between the ions and the surface (which could be different for e.g. $\mathrm{Na}^{+}$and $\mathrm{Cs}^{+}$), as described here by the distribution constant $K_{d}$, tune the effective charge, and hence the magnitude of the electrical (non-specific) interactions, which in turn controls the whole shape of the density profile.

One can express the concentration at the charged surface $c_{0}(L / 2)$ as a function of the total amount of mobile ions in the interlayer, by integrating (5). Since at equilibrium we furthermore have $k_{1}^{\prime} c_{0}(L / 2)=k_{2} \Gamma_{0}$, we can use these two relations to link $K_{d}$ to the kinetic constants, with the result:

$$
\frac{2 k_{1}^{\prime}}{L k_{2}}=K_{d} \frac{\sin \alpha L}{\alpha L}=K_{d} \frac{2 \pi L_{B} L^{2} \tilde{C}_{0}}{(\alpha L)^{2}+\left(\pi L_{B} L^{2} \tilde{C}_{0}\right)^{2}}
$$

Where one must not forget that $\alpha L$ implicitly depends on $K_{d}$. $\tilde{C}_{0}$ denotes the average concentration in the interlayer; using (6), we have:

$$
\tilde{C}_{0}=\frac{\alpha \tan (\alpha L / 2)}{\pi L_{B} L}=\frac{2 \sigma}{L z}
$$

In the limit of weak electrostatic interactions $(\alpha L \rightarrow 0)$, either because the bare charge density is small, or because the adsorption is strong $\left(K_{d} \gg 1\right)$-resulting in a screening of the interactions- or finally because the interlayer spacing is very small (see (8)), the concentration profile becomes very "flat", and almost equal to its average value $\tilde{C}_{0}$. In this case one simply has $\alpha^{2}=2 \pi L_{B} \tilde{C}_{0}$, as expected in the Debye-Hückel limit. Moreover, in this limit equation (9) simplifies to $K_{d}=2 k_{1}^{\prime} / k_{2} L$.

The limitations of the Poisson-Boltzmann equation[29] arise from the fact that it neglects ionic correlations beyond a mean-field treatment of the electrostatic interactions. In particular, the ionic size, which is a fundamental parameter for the description of ion transport in bulk electrolyte solutions[30], is neglected. For low to moderate charge lamellar systems such as clays[31, 32], the hard sphere repulsion between the ions adds 
small oscillations to the Poisson-Boltzmann concentration profile, as can be shown from continuous solvent Monte-Carlo simulations. Yet the latter do not correspond to the oscillations seen in discrete solvent molecular dynamics simulations[19]. In that case the ionic concentration profile still oscillates around the PB curve but the period of the oscillations is different: they are in fact reminiscent of the layering of the solvent. Such an effect could be approximately taken into account by adding an oscillating term to the external potential experienced by the ions. The magnitude of such oscillations is not large, though. Therefore, the Poisson-Boltzmann treatment is probably not too bad an approximation, at least for low to moderate charge surfaces and large interlayer distances.

\section{Steady-state polarization - Permittivity}

In dielectric spectroscopy measurements, an alternating electric field of small magnitude is applied to the sample, at a given frequency $\omega$. Ions move under the applied field, but are stopped by the clay sheets. This is strictly analogous to the situation of an electrolyte between two blocking electrodes, excepted that the only ions present in our case are the counter-ions compensating the charge of the surfaces. This motion leads to an excess of ions at the surface (and a lack at the other surface), so that the local chemical equilibrium is no longer satisfied.

After a certain time, a steady state is reached, and all physical quantities evolve with an angular frequency $\omega$, possibly with a phase lag with respect to the applied field $E_{\text {app }}$. In a typical experiment, the frequencies range from a few $\mathrm{Hz}$ to a few $\mathrm{GHz}\left(10^{9} \mathrm{~Hz}\right)$, so that the propagation time of the electromagnetic interactions $L / c \simeq 10^{-16} s$ (for $L$ of a few hundreds of $\mathrm{nm}$ ) is negligible compared to the period of the oscillations of the electric field. For higher frequencies, which are experimentally accessible, one starts to

probe the solvent relaxation, in which we are not interested here. The approximation of quasi-stationary state is therefore perfectly justified for computing the electric field.

The presence of the electric field breaks the symmetry with respect to the interlayer 
mid-plane, and an electrical polarization appears. That is precisely this polarization, which also evolves with the frequency $\omega$, that is measured. For sufficiently weak applied fields, $P(\omega)$ is proportional to $E_{a p p}$, which allows to define the dielectric constant $\epsilon_{\mathrm{r}}$ by

$$
P(\omega)=\epsilon_{0} \epsilon_{\mathrm{r}} E_{\text {app }}(\omega)
$$

It is commonly split into real and imaginary parts as $\epsilon_{\mathrm{r}}=\epsilon_{\mathrm{r}}^{\prime}-i \epsilon_{\mathrm{r}}^{\prime \prime}\left(\right.$ where $i^{2}=-1$ ). The rate of absorption of energy at a given frequency is proportional to $\epsilon_{\mathrm{r}}^{\prime \prime}$.

We now calculate the polarization as a function of frequency, in the linear response regime. The excess quantities (above $c_{0}, E_{0}$ and $\Gamma_{0}$ ), are denoted by $c_{1}, E_{1}$ and $\delta \Gamma$. In complex notation, for a quantity $f(x, t)$ we have:

$$
f(\omega)=f_{0}(x)+f_{1}(x) e^{i \omega t}
$$

the reference value $f_{0}$ being zero for $P, E_{a p p}, \delta \Gamma$ and $j$, while it is given by (5) and (7) for $c$ and $E$; for $\Gamma_{ \pm}$it is simply $\Gamma_{0}$. One can show that (when the steady state is reached) $c_{1}$ is an odd function of $x$, while $E_{1}$ and $j$ are even, and $\delta \Gamma_{+}=-\delta \Gamma_{-}$. Furthermore, $c_{1}, E_{1}$, $\delta \Gamma, j$ and $P$ are all proportional to $E_{a p p}$ (first order terms). Higher order terms have to be left aside in calculating the permittivity (linear response).

The polarization is the first moment of the charge distribution. In computing the latter, one must not forget the contribution of the charge excess at the surfaces:

$$
P=z \frac{\delta \Gamma_{+}-\delta \Gamma_{-}}{2}+\frac{z}{L} \int_{-L / 2}^{L / 2} x c_{1}(x) \mathrm{d} x
$$

Using (4) and the expression of the internal electric field at the boundaries, $E_{1}( \pm L / 2)=$ $\mp z \delta \Gamma_{ \pm} / \epsilon_{0} \epsilon_{s}$, one simply finds after integrating by parts:

$$
P=-\frac{\epsilon_{0} \epsilon_{s}}{L} \int_{-L / 2}^{L / 2} E_{1}(x) \mathrm{d} x
$$

The flux of counter-ions up to first order in $E_{a p p}$ is (the zeroth order term vanishes):

$$
j(x)=-D \frac{\mathrm{d} c_{1}}{\mathrm{~d} x}+\frac{z D}{k_{B} T}\left[c_{0}(x) E_{1}(x)+c_{1}(x) E_{0}(x)+c_{0}(x) E_{a p p}\right]
$$


Conservation of matter in the interlayer reads in complex notation:

$$
i \omega c_{1}(x)=-\frac{\mathrm{d} j}{\mathrm{~d} x}
$$

Combining (15) and (16) one obtains a differential equation satisfied by $E_{1}\left(c_{1}\right.$ is its derivative, modulo a proportionality constant $z / \epsilon_{0} \epsilon_{s}$, see equation (4)). One then replaces $c_{0}$ and $E_{0}$ by their expressions (5) and (7):

$$
\frac{1}{\alpha^{2}} \frac{\mathrm{d}^{2} E_{1}}{\mathrm{~d} x^{2}}-\frac{2}{\alpha} \tan (\alpha x) \frac{\mathrm{d} E_{1}}{\mathrm{~d} x}-\left[\frac{i \omega}{D \alpha^{2}}+\frac{2}{\cos ^{2}(\alpha x)}\right] E_{1}(x)=\frac{2}{\cos ^{2}(\alpha x)} E_{a p p}
$$

The characteristic time that appears in (17), $\tau_{D}=1 / D \alpha^{2}$, is analogous to the Debye relaxation time in bulk electrolytes, and depends on the distribution constant $K_{d}$ (because $\alpha$ does). It is conveniently expressed as a function of the diffusion time $\tau_{\text {diff }}=L^{2} / D$ which an ion takes to diffuse over the interlayer spacing $L$. One simply has:

$$
\tau_{D}=\frac{\tau_{\text {diff }}}{(\alpha L)^{2}}
$$

The differential equation is solved using the parity of $E_{1}$, together with the boundary condition satisfied by the evolution of the adsorption:

$$
i \omega \delta \Gamma_{+}=k_{1}^{\prime} c_{1}(L / 2)-k_{2} \delta \Gamma_{+}
$$

or introducing the dimensionless parameter $\mu=\tau_{\chi} / \tau_{\text {diff }}$ :

$$
\frac{1}{K_{d}}\left[1+i \omega \tau_{\text {diff }} \frac{\mu}{(\alpha L)^{2}}\left(1+\frac{K_{d}}{2} \frac{\sin \alpha L}{\alpha L}\right)\right] \delta \Gamma_{+}=\frac{\sin \alpha L}{2 \alpha L} \times L c_{1}(L / 2)
$$

where one has also used (1) and (9). $\mu$ characterizes the relative rates of chemical relaxation and diffusion: when $\mu \ll 1$ chemistry is "fast", and the density relaxation is dominated by diffusion (over the shortest distance between the screening length $1 / \alpha$ and the interlayer spacing $L$ ), while $\mu \gg 1$ corresponds to a relaxation of the density which is dominated by the chemical reaction at the surfaces.

After some algebra, detailed in Appendix A, one finds for the excess internal electric 
field:

$$
\left\{\begin{array}{l}
E_{1}(x)=-\frac{D \alpha^{2}}{i \omega} E_{a p p}\left[\frac{1}{\cos ^{2}(\alpha x)}-\xi(\omega) g(\alpha x)\right] \\
g(u)=\frac{(\gamma-1) \cos [(\gamma+1) u]+(\gamma+1) \cos [(\gamma-1) u]}{\cos ^{2} u} \\
\gamma=\sqrt{1-\frac{i \omega}{D \alpha^{2}}}=\sqrt{1-i \omega \tau_{D}}=\sqrt{1-\frac{i \omega \tau_{\mathrm{diff}}}{(\alpha L)^{2}}} \\
\xi(\omega)=\frac{\chi(\omega)}{\cos ^{2}(\alpha L / 2)\left[\chi(\omega) g(\alpha L / 2)+i \omega \tau_{D} \sin (\gamma \alpha L / 2) \sin (\alpha L / 2)\right]} \\
\chi(\omega)=\sin ^{2}(\alpha L / 2)+\frac{1}{K_{d}}\left[1+i \omega \tau_{\chi} \frac{1}{(\alpha L)^{2}}\left(1+\frac{K_{d}}{2} \frac{\sin \alpha L}{\alpha L}\right)\right]
\end{array}\right.
$$

The excess concentration $c_{1}$ can then be obtained by equation (4), but it is not necessary for computing the polarization. The frequency dependence of $\gamma$ is reminiscent of that, unusual, of the Debye-Falkenhagen relaxation effect in bulk electrolytes[4]. Inserting (21) in (14), we get the polarization:

$$
P(\omega)=\epsilon_{0} \epsilon_{s} E_{a p p} \frac{1}{i \omega \tau_{D}}\left[\frac{\tan (\alpha L / 2)}{\alpha L / 2}-\xi(\omega) \frac{1}{\alpha L} \int_{-1}^{1} g(u) \mathrm{d} u\right]
$$

The last integral can be performed analytically, which gives the final result for the dielectric constant $\epsilon_{\mathrm{r}}$ :

$$
\epsilon_{\mathrm{r}}(\omega)=\epsilon_{s} \frac{1}{i \omega \tau_{D}} \frac{\tan (\alpha L / 2)}{\alpha L / 2}\left[1-2 \xi(\omega) \frac{\sin \gamma \alpha L / 2}{\sin \alpha L / 2}\right]
$$

Equations (21) and (23) constitute the major findings of the present article, and will be the starting point of the discussion of the influence of the various parameters. We would like to underline here the fact that the (relative) simplicity of the solution, and the existence itself of a completely analytical solution were not obvious at all, considering the non-linearity of the problem.

\section{Discussion}

The frequency dependence of the dielectric constant is not trivial: it results from an interplay between electrical relaxation and chemical relaxation at the charged surfaces. Chemistry is present in (23) only through the parameter $\xi(\omega)$ given by (21), which also 
depends on the electrostatics, via the dimensionless parameter $\alpha L$. The frequency dependence of $\xi(\omega)$ is then incorporated in expression (23) which depends apart from $\xi$ only on electrostatics. It is therefore quite difficult to have a clear feeling for what is the exact behavior of the complex permittivity, and one will have to compute it numerically as a function of frequency in order to gain insight in the influence of the thermodynamics $\left(K_{d}\right)$ and on the dynamics (chemical vs. electrical relaxation rate, i.e. the value of $\mu$ ) on the complex permittivity.

Interestingly, some important limits can be studied analytically. Among them, the static permittivity is of particular interest. It is defined as the low frequency limit of (23), and it can be shown to be real and finite, which is not obvious from equation (23). The result is simply:

$$
\epsilon_{\mathrm{r}}(\omega \rightarrow 0)=\frac{\epsilon_{s}}{2}\left[1-\frac{\tan (\alpha L / 2)}{\alpha L / 2} \times \frac{\cos ^{2}(\alpha L / 2)}{1+K_{d} \sin ^{2}(\alpha L / 2)}\right]
$$

It is easily checked that the right hand side of equation (24) is positive, whatever the values of $K_{d}$ and $\alpha L$. Two remarks can be made at that point. First, it does not depend on the chemical relaxation time $\tau_{\chi}$, but only on the distribution constant $K_{d}$, nor does it depend on the electrical relaxation time $\tau_{D}=1 / D \alpha^{2}$, but only on the dimensionless structural parameter $\alpha L$. Indeed, when the applied field varies with an infinitely low frequency, all relaxation phenomena (chemical and electrical) occur comparatively very fast, and all information about their dynamics becomes irrelevant. Second, equation (24) implies that the contribution of ionic motion perpendicular to the charged surface is systematically less important than (half of) that of the solvent. This very important point will need to be discussed when turning to the comparison with experiments. We would like to draw the reader's attention to the following point: since equation (24) gives only the ionic contribution to the static permittivity, the solvent contribution $\epsilon_{S}$ should be added to give the full static permittivity of the interlayer, which satisfies from the previous discussion: $\epsilon_{s} \leq \epsilon_{\text {interlayer }} \leq 1.5 \epsilon_{s}$.

The second important limit is that of "slow chemistry". This limit corresponds to 
an infinitely long chemical relaxation time, and for a fixed $K_{d}$ (fixed effective charge), it implies that both forward (adsorption) and backward (desorption) reactions are extremely slow. The case of no chemical exchange at all is therefore encompassed in this limit $(\mu \rightarrow \infty)$. The parameter $\xi(\omega)$ from equation $(21)$ simply becomes:

$$
\frac{1}{\xi(\omega)}=\cos ^{2} \frac{\alpha L}{2} g\left(\frac{\alpha L}{2}\right)=2 \gamma \cos \frac{\gamma \alpha L}{2} \cos \frac{\alpha L}{2}+2 \sin \frac{\gamma \alpha L}{2} \sin \frac{\alpha L}{2}
$$

The dielectric constant then reduces to:

$$
\epsilon_{\mathrm{r}}(\omega)=\frac{2}{\alpha L} \frac{1}{i \omega \tau_{D}} \times \frac{\gamma \tan (\alpha L / 2)-\tan (\gamma \alpha L / 2)}{\gamma+\tan (\alpha L / 2) \tan (\gamma \alpha L / 2)}
$$

where $\gamma$ is still given by (21). The polarizability of the interlayer, as described by its complex permittivity $\left(\epsilon_{\mathrm{r}}=\epsilon_{\mathrm{r}}^{\prime}-i \epsilon_{\mathrm{r}}^{\prime \prime}\right)$ depends greatly on $\alpha L$, or equivalently on $L / \lambda$, as can be seen from figure 3 , where $\epsilon_{\mathrm{r}}^{\prime \prime} / \epsilon_{S}$ has been plotted as a function of frequency $\left(\omega \tau_{\text {diff }}\right)$ and of $L / \lambda$ (both in logarithmic scales). Within this representation, the usual Bode diagrams $\left(\epsilon_{\mathrm{r}}^{\prime \prime}\right.$ vs. $\left.\log \omega\right)$ are found along horizontal lines. There is no additional information contained in $\epsilon_{\mathrm{r}}^{\prime}(\omega)$, because it can be directly deduced from $\epsilon_{\mathrm{r}}^{\prime \prime}(\omega)$ via the Kramers-Kronig relations. Each relaxation appears on the Bode diagram for the imaginary part as a peak at the corresponding frequency.

For small $\alpha L$, the equilibrium profile is flat, and is not very sensitive to an applied field. Indeed, the permittivity is then:

$$
\epsilon_{\mathrm{r}}(\omega) \stackrel{\alpha L \rightarrow 0}{\sim} \frac{(\alpha L)^{2}}{i \omega \tau_{\text {diff }}}\left[1-\frac{\tan \left(\sqrt{-i \omega \tau_{\text {diff }}} / 2\right)}{\sqrt{-i \omega \tau_{\text {diff }}} / 2}\right]
$$

and vanishes as $(\alpha L)^{2}$. One can show that the absorption peak (maximum of $\epsilon_{\mathrm{r}}^{\prime \prime}(\omega)$ ) is located in this limit for $\omega \tau_{\text {diff }} \approx \pi^{2}$ (red curve of figure 3 ), independently of $\alpha L$, because for low charge densities, the relevant distance is not the screening length but rather the interlayer spacing; hence the relevant timescale is not $\tau_{D}$ but $\tau_{\text {diff }}$.

At the other extreme (of charge saturation), the ionic distribution is sharply peaked in the vicinity of the charged surfaces. The profile is deformed more easily by an applied field. The permittivity is in this limit:

$$
\epsilon_{\mathrm{r}}(\omega) \stackrel{\alpha L \rightarrow \pi}{\sim} \frac{2}{i \omega \tau_{\text {diff }}} \frac{\sqrt{\pi^{2}-i \omega \tau_{\text {diff }}}}{\tan \left(\sqrt{\pi^{2}-i \omega \tau_{\text {diff }}} / 2\right)}
$$


and is finite. The absorption peak is located at $\omega \tau_{\text {diff }} \approx \pi^{3}$, and is seen on figure 3 (green curve) to coincide at large $\alpha L$ with $\omega \tau_{D} \approx \pi$ (blue curve). The relevant timescale is then the diffusion time over the screening length.

Therefore for the case of no exchange, the dynamics of the charge density is entirely determined by its structure $(L / \lambda)$, and corresponds to the diffusion of the ions over the relevant length scale, which can be either the interlayer distance or the screening length, depending on the strength of the electrostatic interactions.

The last noteworthy limit is that of fast chemical exchange $(\mu \rightarrow 0)$. In equation $(21)$, $\xi(\omega)$ simplifies to:

$$
\frac{1}{\xi(\omega)}=\cos ^{2} \frac{\alpha L}{2} g\left(\frac{\alpha L}{2}\right)+i \omega \tau_{D} \sin \frac{\gamma \alpha L}{2} \sin \frac{\alpha L}{2} \times \frac{K_{d} \cos ^{2} \alpha L / 2}{1+K_{d} \sin ^{2} \alpha L / 2}
$$

In this limit too the chemical relaxation time has disappeared, and the only relevant timescale corresponds to the transport of mobile ions. Further simplifications are obtained in the weak and strong electrostatics limits. In the former, $\epsilon_{\mathrm{r}}(\omega)$ also vanishes as $(\alpha L)^{2}$ :

$$
\epsilon_{\mathrm{r}}(\omega) \stackrel{\alpha L \rightarrow 0}{\sim} \frac{(\alpha L)^{2}}{i \omega \tau_{\text {diff }}}\left[1-\frac{\tan \left(\sqrt{-i \omega \tau_{\text {diff }}} / 2\right)}{\sqrt{-i \omega \tau_{\text {diff }}} / 2} \times \frac{1}{1+\frac{K_{d} \sqrt{-i \omega \tau_{\text {diff }}}}{4} \tan \left(\sqrt{-i \omega \tau_{\text {diff }}} / 2\right)}\right]
$$

The position of the absorption peak now depends on $K_{d}$, but for weak adsorption $\left(K_{d} \ll\right.$ 1), one recovers the same result as in the slow exchange limit (equation (27)). This is because in the weak adsorption case, what happens at the surfaces is of secondary importance with respect to the transport of the mobile ions; in particular, the rate of the exchange does not significantly affects the overall dynamics. In the fast exchange, strong electrostatics limit $(\alpha L \rightarrow \pi)$, one finds the same result as in the slow exchange, strong electrostatics limit (equation (28)).

We now turn to the more complex case where the chemical reaction takes place at a finite rate. The relaxation dynamics of the charge density now involves an interplay between chemical reaction at the surfaces and transport in the interlayer. In order to study simultaneously the influence of the chemical rate (or $\mu=\tau_{\chi} / \tau_{\text {diff }}$ ) and of the relative 
abundance of bound/free ions $\left(K_{d}\right)$, we have computed numerically the absorption spectrum (Bode diagram for $\epsilon_{\mathrm{r}}^{\prime \prime}$ ) for a fixed surface charge density and fixed interlayer distance $\left(\sigma_{0}=0.131 \mathrm{Cm}^{-2}, L=6 \AA\right)$ for univalent ions in a solvent of dielectric constant $\epsilon_{S}=78$. These values were already used to illustrate the equilibrium profiles in section 2 (see figure $2 \mathrm{~b})$. As the affinity of the ions for the surface $\left(K_{d}\right)$ increases, the effective charge of the surfaces decreases, resulting in weaker electrostatic interactions ( $\alpha L$ decreases).

The results are reported in figure 4 for $K_{d}=0.1$ (a), 1.0 (b) and 10 (c), where $\epsilon_{\mathrm{r}}^{\prime \prime} / \epsilon_{S}$ is represented as a function of $\log \left(\omega \tau_{\text {diff }}\right)$ and $\log \mu$. For each exchange rate, the usual Bode diagram is found along a horizontal line. Fast exchange is found at the bottom of each plot, while slow exchange is located at the top. It is obvious from figure 4 that whatever the adsorption, two relaxations are found when the exchange is slow (though with varying relative weights), and only one is left when the chemical exchange rate is very high. This feature is ubiquitous in spectroscopy: although the physical relaxations are different from the present case, it has long been used in Nuclear Magnetic Resonance to evaluate kinetic constants[33, 34, 35].

For slow exchange $(\mu \gg 1)$, the dominant mechanism relaxation is diffusion for weak adsorption (4a), and exchange for strong adsorption (4c); for intermediate values of the distribution constant, both mechanisms give a significant contribution to the permittivity (4b). Indeed, the maximum of $\epsilon_{\mathrm{r}}^{\prime \prime}$ is found not to depend on $\mu$ in this limit for the weak adsorption case. The secondary peak, which does depend on $\mu$ and corresponds to the chemical exchange, contributes to less than half of the former. The opposite trend is observed for strong adsorption (4c), the primary peak occurring at a frequency proportional to $1 / \tau_{\chi}$.

For fast exchange $(\mu \ll 1)$, the chemical reaction is diffusion limited, and the relaxation is governed by transport only. The position of the maximum in this limit is seen to depend on $K_{d}$, as was explained before, and to be located at higher frequencies for smaller $K_{d}$ 's. For $K_{d}=0.1(4 \mathrm{a})$, the relaxation frequency does not depend on $\mu$, and therefore it coincides with that in the slow chemistry limit. This is because for this choice of the 
numerical parameters, we are in the range of "weak electrostatics" even for small $K_{d}$. Hence, in the fast exchange limit, the permittivity is given by equation (30), which was said to reduce to equation (27) for weak adsorption (small $K_{d}$ ). These numerical results therefore illustrate the announced limiting expressions.

It appears from the preceding discussion that both the thermodynamics $\left(K_{d}\right)$ and the dynamics $\left(\tau_{\chi}\right)$ of the adsorption / desorption processes have a significant influence on the dielectric permittivity of the interlayer. Therefore, one could in principle extract from experimental data the values of these parameters. Indeed, structural informations (bare charge, interlayer spacing, valency of the ions) are generally known from other experimental techniques (e.g. titration, X-ray diffraction), and one could deduce from dielectric spectroscopy measurements the remaining unknown parameters $\left(K_{d}, \tau_{\chi}, \tau_{\text {diff }}\right)$ by fitting the data with equation (23). This promising method suffers however from several limitations, which we now wish to discuss. Those limitations are of two kinds: theoretical on the one hand, and related to the experiments on the other hand.

Let us first focus on limitations of the theory exposed in the present article. The present treatment of the interlayer solution, and particularly of the solvent as a continuum, is valid for not too small interlayer distances. It is expected to break down for distances comparable to the water molecular size, but has been shown to give reasonable results for the density profiles for distances as short as $15 \AA[19]$. At shorter distances, the molecular character of the solvent and of the ions must be included to account for the oscillatory density profiles in the interlayer[36]; a complete description of the dynamical response in this case in order to calculate the dielectric permittivity would be a great challenge. In this respect, the numerical values chosen here to illustrate our discussion might be at the limit of validity of the present theory. However, the results obtained with another system with a larger interlayer distance and a smaller charge density (leaving their product $L / \lambda$ unchanged), would be the same, and fully in the range of applicability of the model. This could easily be obtained e.g. with a less charged clay at higher water content. Therefore the model might not be applicable to the experimental situation that guided us in the 
choice of the numerical values of the parameters, but that does not rule out the validity of the theory. In the same spirit, the choice of the value of the dielectric constant $\epsilon_{S}$ is not straightforward, since for small (a few molecular) layers, its value (if its very existence is admitted in that case) might be different from its bulk one. Under confinement down to a few $\AA$, it has been shown that the dielectric constant of water has generally a lower value than in the bulk [37]. Thus, the results are expected to compare favorably with experiments only for sufficiently large interlayer distances, for which these problems are ruled out.

In order to compare with experimental data, one should be able to take into account the contribution of the lamellae, which were assumed to be charged planes in the present description. This simplification allowed each interlayer spacing to be treated independently. In real systems however, the finite extent of the lamellae, and their possible dielectric properties might give a contribution to the dielectric response of the whole system. In particular, a mismatch between the dielectric constant of the solvent and that of the lamellae would necessitate to take all the interactions with image charges into account[38]. These limitations are probably not too serious. First, assuming that each cell is independent of the others is valid as soon as the surfaces are charged enough, which results in a screening of the electrical interactions across the layers. Second, their dielectric properties can be considered -at least for inorganic lamellae (such as clays)- as frequency independent, in this frequency region ( $\mathrm{Hz}$ to $\mathrm{GHz})$, because electronic polarization occurs at much higher frequencies. Thus the contribution of the layers reduces to a single dielectric constant $\epsilon_{L}$, which can then be introduced in the calculation of the resulting dielectric permittivity of the lamellar system.

Finally, two difficulties arise not from the model, but from its predictions. As was mentioned earlier, the ionic contribution to the complex permittivity is less than half of that of the solvent (see equation (24)). This could make it difficult to separate from that of the solvent, although the latter occurs at higher frequencies. The other point is that in some cases (and only in these case), the predicted permittivity is not very sensitive to 
the value of the parameters. For example, close to the charge saturation $(\alpha L \rightarrow \pi)$, the value of $\alpha L$ depends only little on the exact value of $L / \lambda$. Hence, extracting the latter from the former cannot be achieved with a great precision. Similarly, when the chemical exchange is either very fast or very slow, the resulting permittivity does not depend on the precise value of $\mu=\tau_{\chi} / \tau_{\text {diff }}$. Therefore, one can hope to extract precise microscopic information only if the system is such that the electrostatics is not too strong $(L / \lambda$ not too large) and the chemical exchange is both not too fast and not too slow ( $\mu$ not too large and not too small). These remarks do not minimize the range of the present model, but only the hope that we can have to deduce microscopic information in some particular cases.

\section{Conclusion}

A fully analytical expression of the complex permittivity of lamellar systems in absence of added salt has been derived. The chemical exchange governing the partition between free and bound ions was properly taken into account, and the influence of both thermodynamic (distribution constant) and kinetic characteristics of this equilibrium was discussed. The main conclusions are twofolds.

First, when chemical exchange is infinitely slow (no exchange, just a static partition between free and bound ions), the only relaxation process is due to transport. The study of the complex permittivity as a function of frequency reveals that depending on the "strength of electrostatics" (the ratio between the interlayer distance $L$ and the Gouy length $\lambda$ ), the relevant length scale vary between the whole interlayer distance (weak interactions case) and the screening length only (strong interactions case). Accordingly, the characteristic relaxation time ranges from the diffusion time (over the interlayer distance) to the Debye relaxation time (diffusion over the screening length $\alpha^{-1}$ ). Furthermore the magnitude of the considered contribution to complex dielectric permittivity also depends greatly on $L / \lambda$. When electrostatic interactions are weak, the ionic profile in the inter- 
layer is flat and not very deformable. Hence, the contribution of normal ionic fluxes to the complex permittivity is small, and other phenomenon must be accounted for. In particular, for small interlayer spacings, the ionic motion along the charged surfaces, which is usually not considered, must be taken into account. This motion is the subject of a forthcoming paper [39].

Second, when the rate of chemical exchange is finite, two mechanisms can give a significant contribution to the complex permittivity: to the diffusion relaxation mode now adds the chemical relaxation mode. The relative weight of both mechanisms depends on the distribution constant $K_{d}$ between bound and free ions, and on the rate of exchange. For slow exchange, relaxation is dominated by transport for weak adsorption and exchange for strong adsorption; for intermediate adsorption both are important. For fast exchange, the reaction at the surface becomes limited by the transport of reactants (free ions) in the interlayer; the characteristic time then depends on $L / \lambda$ (diffusion over $L$ or the screening length $\left.\alpha^{-1}\right)$.

To our knowledge, this is the first time that the complex permittivity of charged lamellar systems without added salt was determined (and this could be done analytically), taking properly into account the chemical exchange at the surfaces. The conclusions of the present article will be useful in the analysis of experimental data on such systems, for which the predictions of the hitherto available models (with added salt) do not hold. We have particularly in mind the study of clay gels (at sufficiently high water content). Further refinements of the model are envisaged. First, the calculations of the permittivity of the whole system, including the contribution of the dielectric layers, in the spirit of Sørensen[12], would allow for a direct comparison with experiments even if the contribution of the latter is not negligible. Second, the generalization to other geometries would open the way for the study of other no added salt cases: the convex spherical case is that of colloidal suspensions, while the concave spherical case would provide a more realistic model of inclusions in rocks. Finally, it would be of particular interest to try to improve the model in order to investigate small interlayer separation; it will be necessary 
to account for the molecular character of the solvent and of the ions, which increases dramatically the complexity of the problem, but simulations have shown that even in that case, the relaxation of the ionic cloud does not differ significantly from the Debye relaxation time calculated with the bulk parameters[40], at least in a certain range of the latter.

\section{Acknowledgments}

B.R. acknowledges financial support of the Ecole Normale Supérieure de Paris.

\section{Appendix A}

In this appendix we derive the solution (21) of the differential equation (17). We need to find an even solution satisfying the appropriate boundary condition (20). We first rewrite (17) using the dimensionless variables $u=\alpha x$ and $\gamma=\sqrt{1-i \omega / D \alpha^{2}}$ as:

$$
f^{\prime \prime}(u)-2 \tan u f^{\prime}(u)-\left[1-\gamma^{2}+\frac{2}{\cos ^{2} u}\right] f(u)=\frac{2}{\cos ^{2} u} E_{a p p}
$$

with $f(u)=E_{1}(x)$. It is easily checked that a particular solution of (31) is:

$$
f_{0}(u)=\frac{1}{\gamma^{2}-1} \frac{E_{a p p}}{\cos ^{2} u}=-\frac{D \alpha^{2}}{i \omega} \frac{E_{a p p}}{\cos ^{2} u}
$$

This solution is an even function. The general solutions can be found by introducing the change of variable $v=\sin u(0 \leq|v| \leq 1)$ and dropping the right hand side of equation (31), leading to:

$$
\left(1-v^{2}\right) h^{\prime \prime}(v)-3 v h^{\prime}(v)-\left[1-\gamma^{2}+\frac{2}{1-v^{2}}\right] h(v)=0
$$


with $h(v)=f(u)$. Two independent solutions for $-1 \leq v \leq 1$ are:

$$
\begin{aligned}
f_{1}(u) & =i \sqrt{2 \pi} \frac{P_{\gamma-1 / 2}^{3 / 2}(u)}{\sqrt{\cos u}} \\
& =\frac{(\gamma-1) \cos [(\gamma+1)(\pi / 2-u)]+(\gamma+1) \cos [(\gamma-1)(\pi / 2-u)]}{\cos ^{2} u} \\
f_{2}(u) & =-i \sqrt{2 \pi} \frac{Q_{\gamma-1 / 2}^{3 / 2}(u)}{\sqrt{\cos u}} \\
& =\frac{(\gamma-1) \sin [(\gamma+1)(\pi / 2-u)]-(\gamma+1) \sin [(\gamma-1)(\pi / 2-u)]}{\cos ^{2} u}
\end{aligned}
$$

where $P$ and $Q$ are associated Legendre functions (see e.g. reference[41]). From these two solutions an even solution can be found by forming the appropriate linear combination, namely:

$$
g(u)=\cos (\pi \gamma / 2) f_{2}(u)-\sin (\pi \gamma / 2) f_{1}(u)
$$

After some basic trigonometric manipulations this simplifies to the expression of $g$ given by (21), which is obviously even.

Therefore, the full solution for $E_{1}(x)$ is of the form given by (21) where the integration constant $\xi(\omega)$ remains to be determined from the boundary conditions. Equation (20) can be rewritten in terms of $E_{1}$ using $E_{1}(L / 2)=-\delta \Gamma_{+} z / \epsilon_{0} \epsilon_{s}$ and $E_{1}^{\prime}(L / 2)=c_{1}(L / 2) z / \epsilon_{0} \epsilon_{s}$ (see equation (4)). Using the analytic form $(21)$ for $E_{1}(x)$, it is then straightforward to derive that $\xi(\omega)$ is given by $(21)$. 


\section{References}

[1] J. C. Maxwell, Treatise on Electricity and Magnetism, 3rd Edition, Clarendon Press, 1881.

[2] K.W. Wagner, Arch. Electrotech. 2, 371 (1914).

[3] R.W. Sillars, Proc. Inst. Electr. Eng. 80, 378 (1937).

[4] P. Debye, H. Falkenhagen, Phys. Zeit. 29, 121 (1928).

[5] A. Chandra, B. Bagchi, J. Chem. Phys 112, 1876 (2000).

[6] J. R. Macdonald, Phys. Rev. 92, 4 (1953).

[7] R. Coelho, Rev. Phys. Appl. 18, 137 (1983).

[8] R. Coelho, J. Non-Cryst. Solids 131-133, 1136 (1991).

[9] T. L. Chelidze, Y. Gueguen, Geophys. J. Int. 137, 1 (1999).

[10] T.S. Sørensen, J. Colloid Interface Sci. 168, 437 (1994).

[11] T.S. Sørensen, V. Compañ, J. Colloid Interface Sci. 178, 186 (1996).

[12] T.S. Sørensen, J. Chem. Soc. Farad. Trans. 93, 4327 (1997).

[13] P.A. Cirkel, J.P.M. van der Ploeg, G.J.M. Koper, Physica A 235, 269 (1997).

[14] C. Chassagne, D. Bedeaux, J.P.M. van der Ploeg, G.J.M. Koper, Colloids Surf. A 210, 137 (2002).

[15] E.M. Trukhan, Sov. Phys. Solid State 4, 2560 (1963).

[16] J. Bisquert, V. Halpern, F. Henn, J. Chem. Phys 122, 151101 (2005).

[17] C. Grosse, J. J. López-García, J. Horno, J. Phys. Chem. B 108, 8397 (2004). 
[18] J. J. López-García, C. Grosse, J. Horno, J. Phys. Chem. B 109, 5808 (2005).

[19] V. Marry, J. F. Dufrêche, M. Jardat, P. Turq, Mol. Phys. 101, 3111 (2003).

[20] J. F. Dufrêche, V. Marry, O. Bernard, P. Turq, Colloids Surf. A 195, 171 (2001).

[21] ANDRA, Référentiel Matériaux, Tome 1: Contexte et objet, Rapport C.RP.AMAT.01.060, 2001.

[22] L. Onsager, S.W. Provencher, J. Am. Chem. Soc. 90, 3134 (1968).

[23] K. Ibuki, N. Nakahara, J. Chem. Phys. 92, 7323 (1990).

[24] P. Porion, M. Al Mukhtar, A. M. Faugère, R. J. M. Pellenq, S. Meyer, A. Delville, J. Phys. Chem. B 107, 4012 (2003).

[25] R. Stannarius, F. Kremer, M. Arndt, Phys. Rev. Lett. 75, 4698 (1995).

[26] N. Nandi, B. Bagchi, J. Phys. Chem. B. 101, 10954 (1997).

[27] C. Baar, R. Buchner, W. Kunz, J. Phys. Chem. B 105, 2906 (2001).

[28] C. Baar, R. Buchner, W. Kunz, J. Phys. Chem. B 105, 2914 (2001).

[29] J.-P. Hansen H. Löwen, Ann. Rev. Phys. Chem. 51, 209 (2000).

[30] J. F. Dufrêche, O. Bernard, S. Durand-Vidal, P. Turq, J. Phys. Chem. $B$ 109, 9873 (2005).

[31] A. Delville P. Laszlo, New J. Chem. 13, 481 (1989).

[32] A. Delville, Langmuir 6, 1289 (1990).

[33] H. S. Gutowsky, C. H. Holm, J. Chem. Phys. 25, 1228 (1956).

[34] E. Grunwald, A. Loewenstein, S. Meiboom, J. Chem. Phys. 27, 630 (1957). 
[35] A. Loewenstein, S. Meiboom, J. Chem. Phys. 27, 1067 (1957).

[36] W. Olivares, B. Sulbarán, M. Lozada-Cassou, J. Chem. Phys. 103, 8179 (1995).

[37] S. Senapati, A. Chandra, J. Phys. Chem. B 105, 5106 (2001).

[38] R. Kuellander, S. MarčElua, J. Chem. Phys. 88, 7138 (1988).

[39] B. Rotenberg, A. Cadéne, J. F. Dufrêche, S. Durand-Vidal, J. C. BADOt, P. Turq, submitted.

[40] F. Grün, M. Jardat, P. Turq, C. Amatore, J. Chem. Phys. 120, 9648 (2004).

[41] M. Abramowitz, I. A. Stegun, Handbook of Mathematical Functions, Dover Publications, Inc., New-York. 


\begin{tabular}{ccc}
\hline$K_{d}$ & $L / \lambda$ & $\alpha L$ \\
\hline 0.1 & 4.14 & 1.74 \\
1.0 & 2.28 & 1.38 \\
10 & 0.41 & 0.63 \\
\hline
\end{tabular}

Table 1: Ratio between the interlayer distance $L$ and the Gouy length $\lambda$ (resp. the screening length $\alpha^{-1}$ ) corresponding to the numerical values used for the discussion (see text and figure 4). 
Figure 1: Schematic view of the interlayer. Ions can be either diffusing in the interlayer, or bound to the charged surfaces. Both forms are in chemical equilibrium, exchanging with rates $k_{1}^{\prime}$ and $k_{2}$ (see text). Adsorption of ions results in an effective charge of the surfaces.

Figure 2: (a) Reduced screening parameter $\alpha L$ vs. the ratio $L / \lambda$ between the interlayer distance and the Gouy length, which characterizes the strength of electrostatics. $L / \lambda$ and hence $\alpha L$ decrease with increasing adsorption $\left(K_{d}\right)$. At large fields, the screening length saturates (limit value $\alpha L=\pi$ ). (b) Concentration profiles corresponding to $\alpha L=0.63$ $(\triangle), 1.38(\circ)$ and $1.74(+)$.

Figure 3: Imaginary part of the reduced complex permittivity $\left(\epsilon^{\prime \prime} / \epsilon_{S}\right)$ as a function of frequency (normalized by the diffusion time $\tau_{\text {diff }}=L^{2} / D$ ), and of the ratio $L / \lambda$ between the interlayer distance and the Gouy length, which controls the strength of electrostatics in the interlayer, in the case of infinitely slow exchange (no exchange). For $L / \lambda \ll 1$, the concentration profile is very flat, and not very sensitive to an applied electric field, while for $L / \lambda \gg 1$, electric interactions result in an ionic distribution sharply peaked at the charged surfaces, and deformable by an electric field. The characteristic time for the relaxation process corresponds in the former case to the diffusion over the interlayer distance $\left(\tau \simeq \tau_{\text {diff }} / \pi^{2}\right.$ - red curve) while in the latter case the relevant distance is the screening length $\alpha^{-1}\left(\tau \simeq \tau_{D} / \pi\right.$ - blue curve) which reaches its saturation value $L / \pi$ when $L / \lambda \gg 1\left(\tau \simeq \tau_{\text {diff }} / \pi^{3}\right.$ - green curve $)$.

Figure 4: Imaginary part of the reduced complex permittivity $\left(\epsilon^{\prime \prime} / \epsilon_{S}\right)$ for $K_{d}=0.1$ (a), 1.0 (b) and 10 (c), as a function of frequency (normalized by the diffusion time

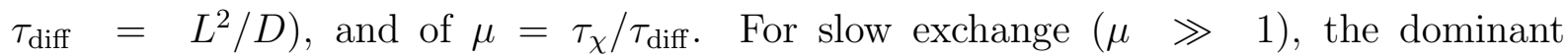
mechanism is diffusion for weak adsorption (a), and exchange for strong adsorption (c); for intermediate values of the distribution constant, both mechanisms give a significant contribution (b). For fast exchange $(\mu \ll 1)$, the relaxation is due to transport only, and the position of the maximum depends on $K_{d}$. 


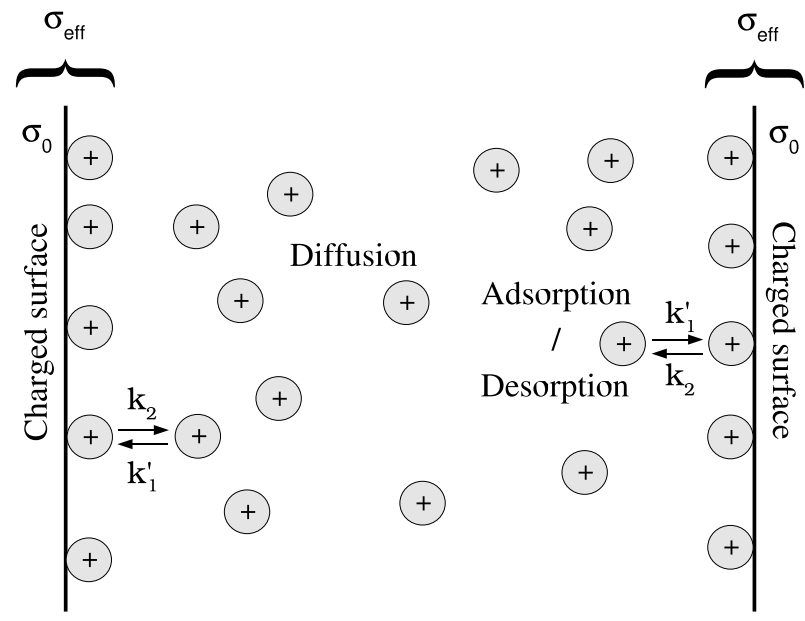

Figure 1: B. Rotenberg et. al 

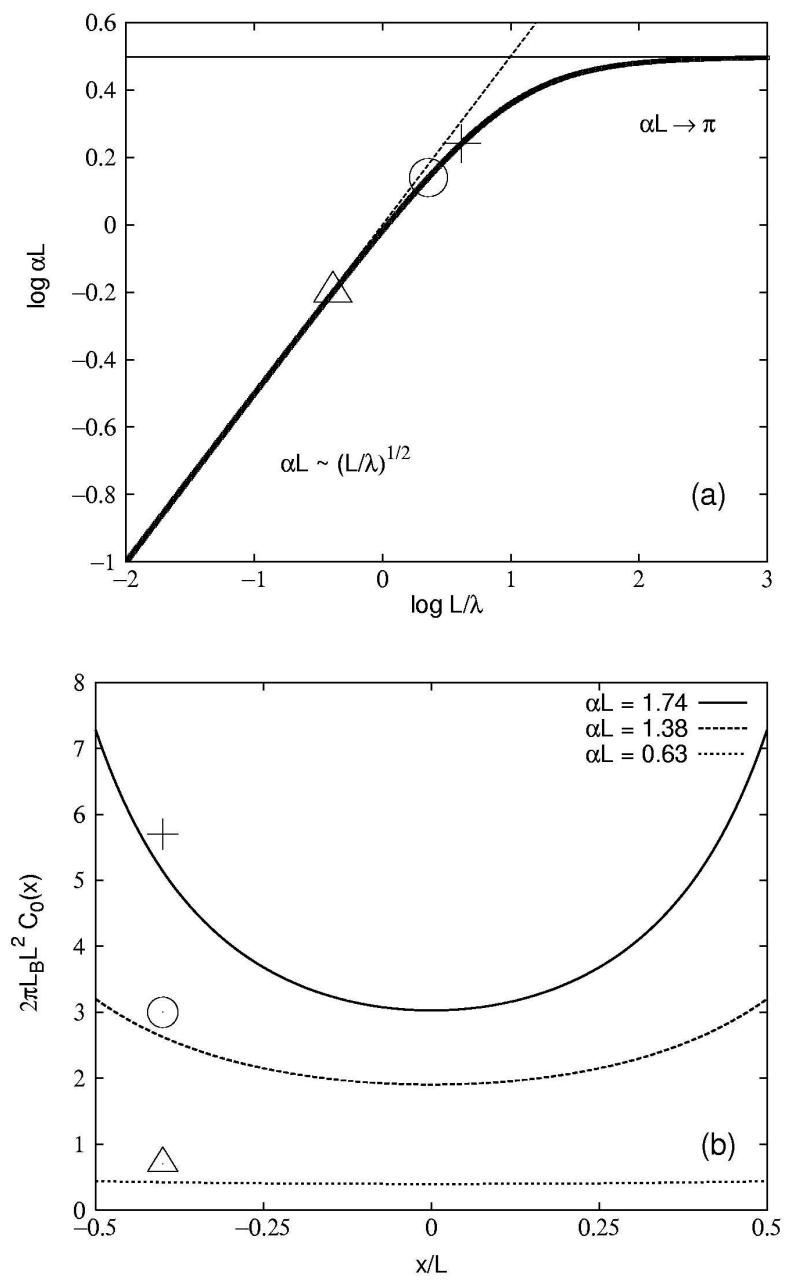

Figure 2: B. Rotenberg et. al 


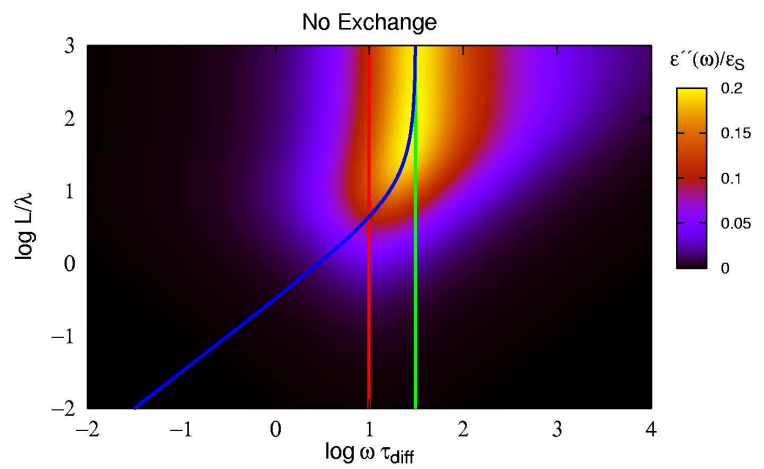

Figure 3: B. Rotenberg et. al 
(a) $K_{d}=0.1$
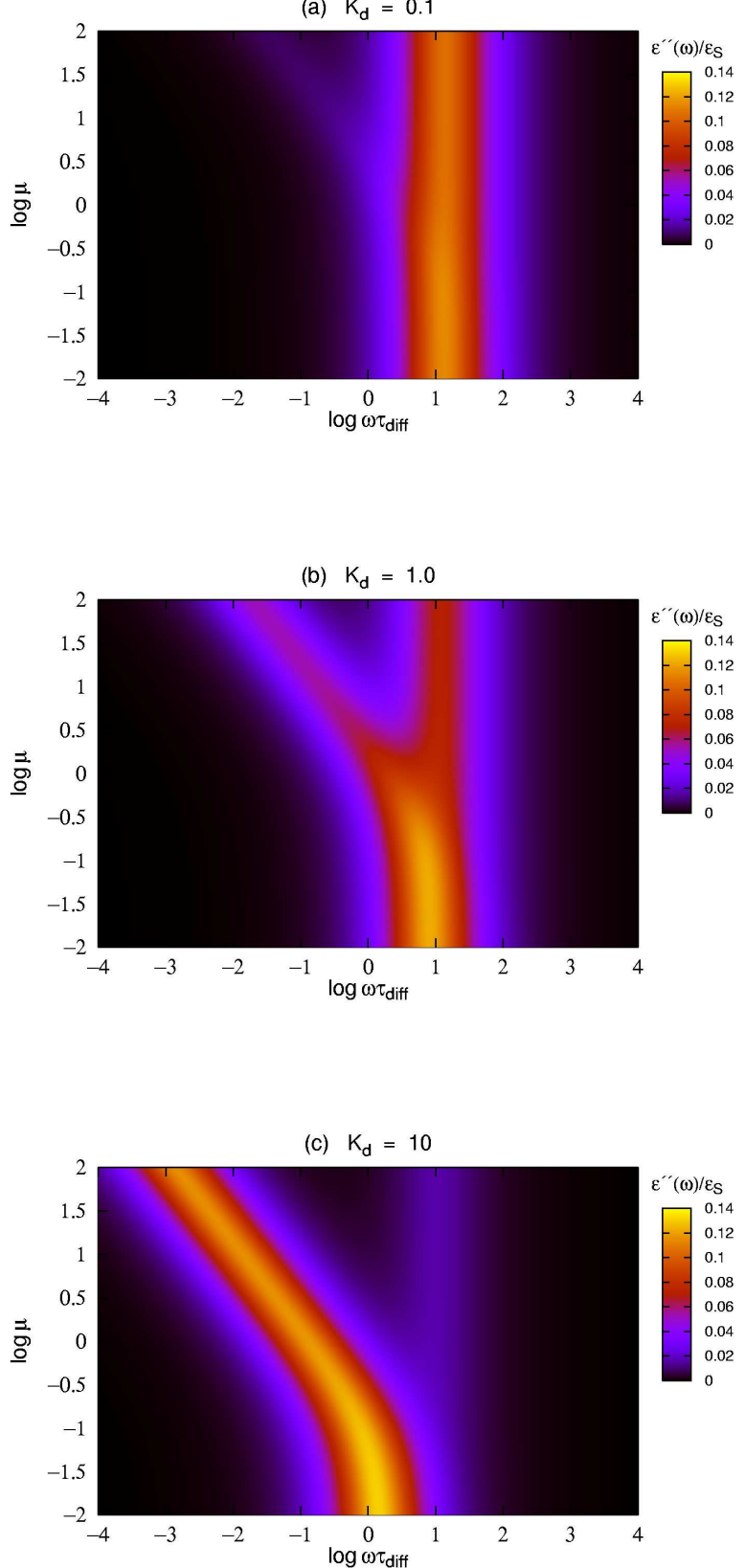

Figure 4: B. Rotenberg et. al 